\title{
A ROBUST CLOCK SYNCHRONIZATION ALGORITHM FOR WIRELESS SENSOR NETWORKS
}

\author{
Jang-Sub Kim, Jaehan Lee, Erchin Serpedin, and Khalid Qaraqe \\ ECE Dept., Texas A\&M University, College Station, TX77843-3128, USA \\ Phone: (979) 8717891 Fax: (979) 8623128 email: serpedin@ece.tamu.edu
}

\begin{abstract}
Recently, the maximum likelihood estimator (MLE) and Cramer-Rao Lower Bound (CRLB) were proposed with the goal of maximizing and assessing the synchronization accuracy in wireless sensor networks (WSNs). Because the network delays may assume any distribution and the performance of MLE is quite sensitive to the distribution of network delays, designing clock synchronization algorithms that are robust to unknown network delay distributions appears as an important problem. By adopting a Bayesian framework, this paper proposes a novel clock synchronization algorithm, called Iterative Gaussian Mixture Kalman Particle Filter (IGMKPF), which is shown to achieve good and robust performance in the presence of unknown network delay distributions. The Posterior Cramer-Rao Bound (PCRB) and the Mean-Square Error (MSE) of IGMKPF are evaluated and shown to exhibit improved performance and robustness relative to MLE.
\end{abstract}

Index Terms - Maximum Likelihood Estimation, State Estimation, Adaptive Filters, Particle Filter

\section{INTRODUCTION}

Clock synchronization between two network nodes is generally accomplished via message exchanges. Due to the presence of non-deterministic and possibly unbounded message delays, messages can get delayed arbitrarily, which makes the clock synchronization very difficult [1]. The most commonly proposed distributions to model the network delays are the Gaussian and exponential probability density functions (pdfs) [2]. The maximum likelihood estimators (MLEs) for clock offset estimation in the presence of symmetric Gaussian and exponential network delays will be referred to as MLEg and MLEe, respectively. In [2], it is shown that MLEg and MLEe are quite sensitive to the network delay distributions. Also, the Cramer-Rao Lower Bound (CRLB) is shown to be inverse proportional to the number of observations [2]. It appears also that to improve the performance, MLEg and MLEe require a larger number of observations. However, since WSNs are power-limited systems, such a solution is not appropriate.
Because of the uncertainties in modeling the network delay distributions, herein we will adopt a particle filtering (PF)based Bayesian approach for estimating the clock offset. The signaling mechanism between the two nodes is the standard two-way message exchange mechanism encountered in protocols such as NTP, TPSN [3]. The PF-method provides an approximate Bayesian solution to the discrete-time recursive optimization problem. Notice that general particle filtering techniques have no optimal proposal distribution and the observation noise density is in general modeled by its first two moments. Therefore, there exists a bias in both the general PF estimator and MLE, and this is due to the finite number of observations. Therefore, MLE and CRLB can not serve as an efficient estimator and tight lower bound, respectively, in the presence of a reduced number of observations. In addition, the PF and its Posterior Cramer-Rao Bound (PCRB) may not be an optimal estimator or serve as a tight lower bound due to the finite number of samples and unknown observation noise density.

To cope with the limitations of MLE and PF, herein we propose the Iterative Gaussian Mixture Kalman Particle Filter (IGMKPF) to estimate the clock offset between two nodes, and analyze the PCRB as a lower bound for its Mean Square Error (MSE) performance. IGMKPF presents a series of features. First of all, IGMKPF is capable of tracking the posterior and observation noise densities in order to reduce the bias which might stem from the observation noise estimation step in the presence of reduced number of observations. If the observation noise density is fully captured and not partially modeled through the information provided by its mean and variance, then the estimator tends to reduce its bias and leads to improved MSE-performance. Second, the IGMKPF estimator deals with non-Gaussian noise efficiently (e.g., by adopting Gaussian Mixture Models (GMMs) to capture various densities). Lastly, as computer simulations illustrate, the optimal estimation settings can be inferred from the analytical lower bound (e.g., PCRB). Computer simulations are conducted to compare the MSE performance of various competitive estimation schemes MLEg, MLEe, IGMKPF, and IGMKPF with perfect network delay noise estimation, with theoretical bounds such as CRLB and PCRB. As a result, when the accuracy of noise distribution estimation is 
improved, the performance of IGMKPF is significantly better than CRLB (and/or ML) in the presence of reduced number of observations. Therefore, the proposed IGMKPF method represents a reliable clock offset estimation scheme fit to overcome the uncertainties caused by the unknown network delay distributions.

The rest of this paper is organized as follows. Section 2 formulates the problem and introduces the state-space clock phase offset estimation model. Due to space limitations, Section 3 presents only the PCRB for the Gaussian model. Section 4 provides a description of the IGMKPF approach for estimating the clock offset in WSNs. The results of computer simulations are given in Section 5. Finally, concluding remarks are presented in Section 6.

\section{PROBLEM FORMULATION AND OBJECTIVES}

The two-way timing message exchange mechanism is a recently proposed clock synchronization scheme for wireless sensor networks [2]. In this mechanism, the synchronization of two nodes $\mathrm{A}$ and $\mathrm{B}$ is achieved through a number of $N$ cycles. Each cycle assumes two message transmissions: one from node $\mathrm{A}$ to node $\mathrm{B}$, followed by a reverse transmission from node $\mathrm{B}$ to node $\mathrm{A}$. At the beginning of the $k$ th cycle, the node $\mathrm{A}$ sends its time reading $T_{1, k}$ to node $\mathrm{B}$, which records the arrival time of the message as $T_{2, k}$, according to its own time scale. Similarly, a time message exchange is performed from node $\mathrm{B}$ to node $\mathrm{A}$. At time $T_{3, k}$, node $\mathrm{B}$ transmits the time information $T_{2, k}$ and $T_{3, k}$ back to node A. Denoting by $T_{4, k}$ the arrival time at node $\mathrm{A}$ of the message sent by node $\mathrm{B}$, node A would then have access to the time information $T_{j, k}$, $j=1, \ldots, 4$ at the end of the $k$ th cycle, which provide sufficient information for estimating the clock phase offset $\theta_{A}$ of node $A$ relative to node $B$ clock.

Similarly to [2], the differences between the $k$ th up and down-link delay observations corresponding to the $k$ th timing message exchange are defined by $U_{k}:=T_{2, k}-T_{1, k}=$ $d+\theta_{A}+L_{k}$ and $V_{k}:=T_{4, k}-T_{3, k}=d-\theta_{A}+M_{k}$, respectively. The fixed value $d$ denotes the fixed (deterministic) propagation delay component (which in general is neglected $(d \approx 0)$ in small range networks that assume RF transmissions). Parameters $L_{k}$ and $M_{k}$ stand for the variable portions of the network delays, and may assume any distribution such as Gaussian, exponential etc..

Given the observation samples $\mathbf{z}_{k}=\left[U_{k}, V_{k}\right]^{T}$, our goal is to find the minimum mean-square error estimate of the unknown clock offset $\theta_{A}$. For convenience, the notation $x_{k}:=$ $\theta_{A}$ will be used henceforth. Thus, it turns out that we need to determine the estimator $\hat{x_{k}}=E\left\{x_{k} \mid \mathbf{z}^{l}\right\}$. Where $\mathbf{z}^{l}$ denotes the set of observed samples up to time $l, \mathbf{z}^{l}=\left\{\mathbf{z}_{0}, \mathbf{z}_{1}, \ldots, \mathbf{z}_{l}\right\}$. Since the clock offset value is assumed to be a constant, the clock offset can be modeled as following the Gauss-Markov model:

$$
x_{k}=F x_{k-1}+v_{k-1},
$$

where $F$ stands for the state transition matrix of the clock offset. The additive process noise component $v_{k}$ can be modeled as Gaussian with zero mean and covariance $E\left[v_{k} v_{k}^{T}\right]=Q=$ $\sigma_{v}^{2}$. The vector observation model is given by [5]:

$$
\mathbf{z}_{k}=\left[U_{k}, V_{k}\right]^{T}=\mathbf{A} d+\mathbf{B} x_{k}+\mathbf{n}_{k},
$$

where $\mathbf{A}=\left[\begin{array}{ll}1 & 1\end{array}\right]^{T}, \mathbf{B}=\left[\begin{array}{ll}1 & -1\end{array}\right]^{T}$, and the observation noise vector $\mathbf{n}_{k}=\left[L_{k}, M_{k}\right]^{T}$ has zero mean and covariance $R=$ $\operatorname{diag}\left\{\sigma_{n}^{2}, \sigma_{n}^{2}\right\}$, and it accounts for the random network delays.

\section{POSTERIOR CRAMER-RAO BOUND FOR SEQUENTIAL BAYESIAN ESTIMATION}

We need a lower bound on the covariance of the estimator, $\hat{x}_{k}$ for the true state $x_{k}$, defined by (1) and (2). Assuming that regularity condition holds for the probability density functions, the posterior Cramer-Rao bound [4] provides a lower bound on the mean-square error matrix for random parameters. Letting $\hat{x}(\mathbf{z})$ denote an estimate of $x$ which is a function of the observations $\mathbf{z}$, the PCRB provides a lower bound on the MSE matrix M, and it is expressed as the inverse of the Bayesian Fischer Information Matrix (BFIM) $J$ :

$$
\mathbf{M}=\mathbf{E}_{\mathbf{z}, \mathbf{x}}\left\{[\hat{\mathbf{x}}(\mathbf{z})-\mathbf{x}][\hat{\mathbf{x}}(\mathbf{z})-\mathbf{x}]^{\mathbf{T}}\right\} \geq \mathbf{J}^{-\mathbf{1}} .
$$

The BFIM for $x$ is defined as $\mathbf{J}=\mathbf{E}_{\mathbf{z}, \mathbf{x}}\left\{-\triangle_{\mathbf{x}}^{\mathbf{x}} \ln \mathbf{p}(\mathbf{z}, \mathbf{x})\right\}$, where $\triangle_{\phi}^{\theta}$ is the $m \times n$ matrix of second-order partial derivatives with respect to the $m \times 1$ parameter vector $\phi$ and $n \times 1$ parameter vector $\theta$. In [4], the BFIM is shown to follow the recursion:

$$
\mathbf{J}_{\mathbf{k}+1}=\mathbf{D}_{\mathbf{k}}^{22}-\left(\mathbf{D}_{\mathbf{k}}^{21}\right)^{\mathbf{T}}\left(\mathbf{J}_{\mathbf{k}}+\mathbf{D}_{\mathbf{k}}^{11}\right)^{-1} \mathbf{D}_{\mathbf{k}}^{12}
$$

where the matrices $\mathbf{D}_{\mathrm{k}}^{\mathrm{ij}}$ are expressed in terms of expectation integrals. Once we have a sample representation of the posterior density, these expectation integrals can be calculated through sample mean approximations. We can obtain the sample-based representation of the posterior pdf $p\left(x_{k+1} \mid \mathbf{z}_{\mathbf{k}+\mathbf{1}}\right)$ by exploiting the work done in particle filtering [6]. Therefore, we can generate weighted samples on a stochastic grid to represent the posterior density and estimate the Fisher component matrices with the empirical averages:

$$
\begin{aligned}
\mathbf{D}_{\mathbf{k}}^{\mathbf{1 1}} & \simeq 1 / N \sum_{n=1}^{N} \Lambda^{11}\left(X_{k}^{(n)}, X_{k+1}^{(n)}\right) \\
\mathbf{D}_{\mathbf{k}}^{\mathbf{1 2}} & \simeq 1 / N \sum_{n=1}^{N} \Lambda^{12}\left(X_{k}^{(n)}, X_{k+1}^{(n)}\right) \\
\mathbf{D}_{\mathbf{k}}^{\mathbf{2 2}} & \simeq 1 / N \sum_{n=1}^{N}\left(\Lambda^{22, a}\left(X_{k}^{(n)}, X_{k+1}^{(n)}\right)\right. \\
& \left.+\Lambda^{22, b}\left(X_{k}^{(n)}, X_{k+1}^{(n)}\right)\right)
\end{aligned}
$$


where $X_{k+1}^{(n)}, n=1, \ldots, N$, are the a posteriori samples representing the density $p\left(x_{k+1} \mid \mathbf{z}_{\mathbf{k}+\mathbf{1}}\right)$ and $N$ stands for the number of samples. The recursion is initialized with

$$
\mathbf{J}_{0}=\mathbf{E}_{\mathbf{x}}\left\{-\triangle_{\mathbf{x}_{0}}^{\mathbf{x}_{0}} \ln \mathbf{p}\left(\mathbf{x}_{\mathbf{0}}\right)\right\} .
$$

\subsection{PCRB for the Gaussian Network Delay Model}

Equation (1) determines the conditional pdf $p\left(x_{k+1} \mid x_{k}\right)$

$$
p\left(x_{k+1} \mid x_{k}\right)=\frac{1}{\sqrt{2 \pi \sigma_{v}^{2}}} e^{-1 / 2 \sigma_{v}^{2}\left[\left(x_{k+1}-x_{k}\right)^{2}\right]},
$$

and (2) decides the conditional pdf $p\left(x_{k+1} \mid \mathbf{z}_{\mathbf{k}+\mathbf{1}}\right)$

$p\left(\mathbf{z}_{\mathbf{k}+\mathbf{1}} \mid \mathbf{x}_{\mathbf{k}+\mathbf{1}}\right)=\frac{1}{\sqrt{\mathbf{2 \pi \sigma _ { \mathbf { n } } ^ { 2 }}}} \mathbf{e}^{-\mathbf{1} / \mathbf{2} \sigma_{\mathbf{n}}^{2}\left[\left(\mathbf{z}_{\mathbf{k}+1}-\mathbf{A d}-\mathbf{B x}_{\mathbf{k}+1}\right)^{2}\right]}$.

Therefore, we can estimate the Fisher component matrices with the empirical averages :

$$
\mathbf{D}_{\mathrm{k}}^{11} \simeq 1 / \sigma_{\mathrm{v}}^{2}, \mathbf{D}_{\mathrm{k}}^{12} \simeq-1 / \sigma_{\mathrm{v}}^{2}, \mathbf{D}_{\mathrm{k}}^{22} \simeq 1 / \sigma_{\mathrm{v}}^{2}+\frac{2}{\sigma_{\mathrm{n}}^{2}} .
$$

From (4), the BFIM for the Gaussian case takes the form:

$$
\mathbf{J}_{\mathrm{k}+1}=\frac{1}{\sigma_{\mathbf{v}}^{2}}+\frac{2}{\sigma_{\mathbf{n}}^{2}}-\left(-1 / \sigma_{\mathrm{v}}^{2}\right)^{\mathrm{T}}\left(\mathbf{J}_{\mathrm{k}}+1 / \sigma_{\mathrm{v}}^{2}\right)^{-1}\left(-1 / \sigma_{\mathbf{v}}^{2}\right) \text {. }
$$

Therefore, from (3), PCRB is the inverse of BFIM $J$. From the above equations, we note that PCRB is a function of $\sigma_{v}^{2}$ and $\sigma_{n}^{2}$. But in [2], the CRLB for ML is expressed as $\operatorname{var}(\hat{x}) \geq \sigma_{n}^{2} / 2 N$. Figs. 1 (a)-(b) shows CRLB and PCRB when the random delay model is Gaussian with zero mean and variance $\sigma_{n}^{2}=1$ for various initializations of the Fisher information matrix $J_{0}$ and different power levels for the process noise $\left(\sigma_{v}^{2}\right)$. The performance depending on the initialization $J_{0}$, PCRB might achieve similar or better performance levels than CRLB [2] depending on how $J_{0}$ is selected. The simulation results illustrated an interesting fact. When the process noise variance is sufficiently small (less than $10^{-4}$ ) and the initial value of Fisher information matrix is $J_{0}=1 /\left(\sigma_{v}^{2}\right)$, PCRLB is significantly lower than CRLB.

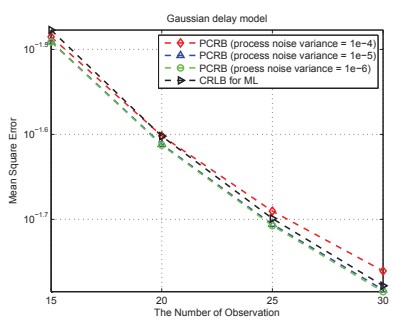

(a) $\sigma_{n}^{2}=1$ and $J_{0}=1$

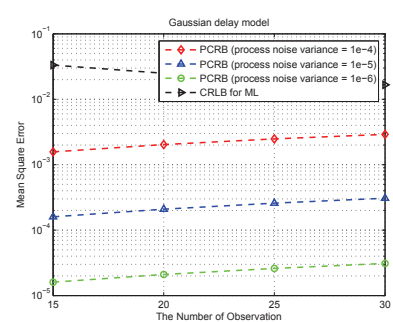

(b) $\sigma_{n}^{2}=1$ and $J_{0}=1 / \sigma_{v}^{2}$
Fig. 1. PCRB and CRLB for symmetric Gaussian delays.

\section{AN ITERATIVE GAUSSIAN MIXTURE KALMAN PARTICLE FILTERING (IGMKPF) APPROACH}

The proposed Iterative Gaussian Mixture Kalman Particle Filtering (IGMKPF) estimator combines the Gaussian mixture Kalman particle filter (GMKPF) [5] with the observation noise density estimator. The observation noise density estimator consists of the state model and a cost function in the form of an innovation equation expressed as the difference between the observation and estimated state posterior pdfs: $p(\mathbf{z})-\mathbf{p}(\hat{\mathbf{z}})$. The innovation equation is produced by considering the estimate yielded by a standard Kalman filter as well as a GMKPF estimator using the prior, process, observation, state posterior, and noise density, which are propagated over time. Fig. 2 provides a perspective on the proposed IGMKPF estimator. In IGMKPF, the first processing stage is represented by the GMKPF which is used to estimate the state posterior density using the observation, prior, and process density. The second processing stage consists in estimating the observation noise density using the innovation, by considering the estimate provided by the standard Kalman filter. The estimated observation noise density, which is used as an input to the first processing stage, is approximated by a GMM fitting function. The iterative process between the two processing stages is repeated up to the end of observations. The pseudo-code of IGMKPF algorithm is next described.

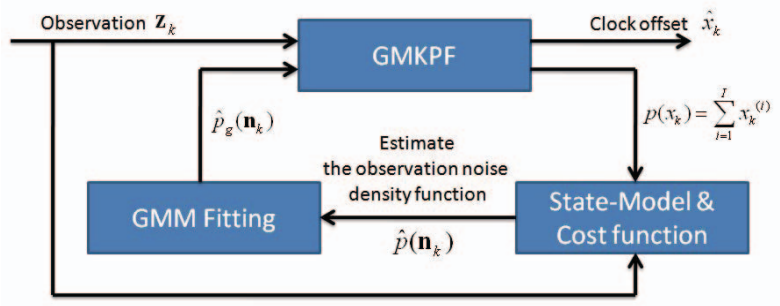

Fig. 2. Block diagram representation of IGMKPF estimator

\section{IGMKPF algorithm}

1. At time $k$, initialize the densities and set the initial state $\hat{x}_{k-1}=x_{M L}$.

2. GMKPF step (estimate the state posterior density)

- Calculate the state posterior density $p_{g}\left(x_{k} \mid \mathbf{z}_{\mathbf{k}}\right)$ using GMKPF.

- If $k$ reaches the end of observations, go to "Infer the conditional mean and covariance step".

3. OND step (estimate the observation noise density)

- Calculate the observation noise density $p(\hat{n})$ given $\mathbf{z}_{\mathbf{k}}$ and $p_{g}\left(x_{k} \mid \mathbf{z}_{\mathbf{k}}\right)$, and state model (eqs. (1) and (2)) 
- The observation noise density using GMM is approximated by:

$$
p_{g}\left(\mathbf{n}_{\mathbf{k}}\right)=\sum_{\mathbf{j}=\mathbf{1}}^{\mathbf{J}} \gamma_{\mathbf{k}}^{(\mathbf{J})} \mathrm{N}\left(\mathbf{n}_{\mathbf{k}} ; \mu_{\mathbf{n}_{\mathbf{k}}}^{(\mathbf{j})}, \mathbf{R}_{\mathbf{k}}^{(\mathbf{j})}\right)
$$

4. $k=k+1$, go to the GMKPF step.

5. Infer the conditional mean and covariance:

$$
\begin{aligned}
& \text { - } \bar{x}_{k}=\sum_{l=1}^{N} w_{k}^{(l)} \chi_{k}^{(l)} \text { and } \bar{P}_{k}=\sum_{l=1}^{N} w_{k}^{(l)}\left(\chi_{k}^{(l)}-\right. \\
& \left.\bar{x}_{k}\right)\left(\chi_{k}^{(l)}-\bar{x}_{k}\right)^{T}
\end{aligned}
$$

\section{SIMULATION RESULTS}

In this section, computer simulations will be conducted to assess the performance of IGMKPF, PCRB-IGMKPF, MLEg [2], MLEe [2], and CRLB for estimating the clock offset in WSNs that are subject to two types of network delays: symmetric Gaussian and exponential. The process noise assumes the power $\sigma_{v}^{2}=10^{-6}$. The number of particles and GMMs are 500 and 3 , respectively. The ML estimators proposed in [2] for symmetric Gaussian and exponential random delays are good examples of initializations. The initial values $\left(\hat{x}_{0}=\right.$ $\hat{x}_{M L E g},\left(\hat{x}_{0}=\hat{x}_{M L E e}\right)$ are near the true values in Gaussian and exponential delay, respectively. Since GMKPF does not initialize the observation noise density and does not track it, its performance is limited. However, IGMKPF tracks the observation noise density using the observation and estimated posterior pdfs. Therefore, the performance of IGMKPF is expected to be better than CRLB.

Figs. 3 (a)-(b) show the MSE of the estimators under the assumption that the random delay models are symmetric Gaussian and exponential, respectively. The notations KN and EN denote the set-ups with known observation noise density and estimated observation noise density, respectively. The MSEs are plotted against the number of observations ranging from 15 to 30 . Note that IGMKPF $(G=3)$ performs much better (over $100 \%$ reduction in MSE) than CRLB and MLEg in the presence of a Gaussian delay model. It is remarkable that the performance of MLEg is proportional to the number of observations, whereas that of IGMKPF is proportional to the number of particles, the number of GMMs, and the accuracy of noise density estimation, but it does not depend on the number of observations [7]. This is a desirable feature for WSNs in order to keep the number of timing exchanges low so that energy is conserved.

\section{CONCLUSIONS}

This paper provided a novel method for estimating the clock offset in wireless sensor networks. The benefits of the proposed synchronization method (IGMKPF) are improved performance compared to CRLB and MLE, and applicability to arbitrary random delay models such as symmetric Gaussian and exponential models. In general, in case of (unknown)

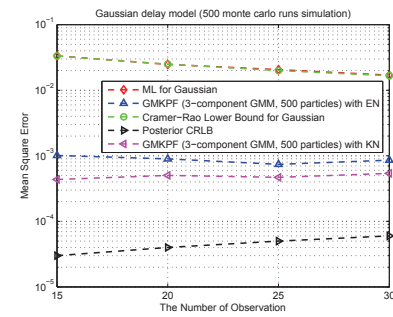

(a) Gaussian $\sigma_{n}^{2}=1$

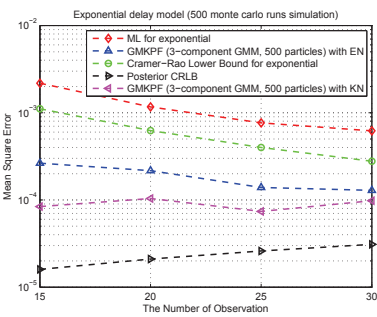

(b) Exponential $\lambda=1$
Fig. 3. MSEs of clock offset estimators.

non-Gaussian distributions, analytical closed-form expressions for MSE-performance do not necessarily exist and it is hard to derive lower bounds. However, this paper derived the posterior Cramer-Rao bound (PCRB) and IGMKPF. An important element in improving the performance of clock estimator is the prediction of unknown observation noise density which led to an improved estimator.

\section{REFERENCES}

[1] I. Akyildiz et al., "Wireless sensor networks: A survey", Computer Networks, vol. 38, no. 4, pp. 393-422, March 2002.

[2] K.-L. Noh, Q. M. Chaudhari, E. Serpedin, and B. W. Suter, "Novel Clock Phase Offset and Skew Estimation Using Two-Way Timing Message Exchanges for Wireless Sensor Networks," IEEE Transactions on Communications, vol. 55, no. 4, April 2007.

[3] I. Skog and P. Handel, "Synchronization by Two-Way Message Exchanges: Cramr-Rao Bounds, Approximate Maximum Likelihood, and Offshore Submarine Positioning," IEEE Transactions on Signal Processing, vol. 58, no. 4, pp. 2351 - 2362, April 2010.

[4] P. Tichavsky, C. Muravchik, A. Nehorai, "Posterior Cramer-Rao Bounds for Discrete-Time Nonlinear Filtering”, IEEE Transactions on Signal Processing, vol. 46, no. 5, May 1998.

[5] J. Kim, J. Lee, E. Serpedin, and K. Qaraqe, "A robust estimation scheme for clock phase offsets in wireless sensor networks in the presence of non-Gaussian random delays," Signal Processing, Elsevier, pp. 11551161, 2009.

[6] A. Doucet, N. de Freitas, N. Gordon, "Sequential Monte Carlo Methods in Practice", Springer-Verlag, 2001.

[7] M. Isard and A. Blake, "CONDENSATION-conditional density propagation for visual tracking”, Int. J. Computer Vision, vol. 29, no. 1, pp. 5-28, 1998. 